\title{
An Attack on SMC-Based Software Protection
}

\author{
Yongdong Wu, Zhigang Zhao, and Tian Wei Chui \\ Institute for Infocomm Research \\ 21, Heng Mui Keng Terrace, Singapore, 119613 \\ \{wydong, zzhao, twchui\}@i2r.a-star.edu.sg
}

\begin{abstract}
Self-modifying codes (SMC) refer to programs that intentionally modify themselves at runtime, causing the runtime code to differ from the static binary representation of the code before execution. Hence SMC is an effective method to obstruct software disassembling. This paper presents a method which circumvents the SMC protection, thus improving the performance of disassembling. By disabling the write privilege to the code section, an access violation exception occurs when an SMC attempts to execute. Intercepting this exception allows the attacker to determine and thus compromise the SMC and generate equivalent static code. Our experiments demonstrate that it is viable and efficient.
\end{abstract}

\section{Introduction}

Currently, most commercial software (e.g., Microsoft ${ }^{\mathrm{TM}}$ Office, Adobe ${ }^{\mathrm{TM}}$ Acrobat) are distributed in binary form to protect the software implementation, particularly mechanisms preventing unauthorized distribution of the software. However, attackers are able to reverse engineer the code in order to analyze and circumvent these protection schemes. For example, the encryption mechanism in Microsoft's Windows Media Player was cracked [1 by reverse engineering, allowing access to protected content in unauthorized environments. Such reverse engineering is heavily dependent on the use of disassembling techniques.

\subsection{Disassembly Technology}

Disassembling aims to produce a higher-level representation of a program to enable comprehension and possible modification to the software. A disassembler enables a cracker to easily translate binary code into human-readable code. For instance, IDAPro [2] translates a binary code into assembly code while Relogix ${ }^{\mathrm{TM}}$ [3] further converts an assembly source into readable, structured, commented $\mathrm{C}$ source - in a truly natural $\mathrm{C}$ style.

Disassembly methods can be distinguished as either static or dynamic disassembly. Static techniques, including linear sweep and recursive traversal, analyze the binary structure statically, parsing the instructions as they are found in the binary image.

Linear sweeping (e.g. objdump [4]) scans the static code from start to end, and decodes the instructions sequentially. Therefore linear sweep disassemblers 
are easy to implement but prone to errors resulting from data bytes that have been interleaved with the code bytes, misleading the disassembler.

Recursive traversal (e.g., IDApro 2, and 5]) follows the control flow of the program, thus avoiding incorrect disassembly of data bytes. However, certain code sections may not be part of the control flow, particularly if the target address is produced in real time (e.g., pointer functions). Hence, the recursive disassembler will not reach and disassemble these regions. To overcome this weakness, a linear sweep algorithm is typically used to analyze these sections.

Dynamic techniques (e.g., rordbg [6]) create a debug environment to run the application. By monitoring the program's execution, a dynamic disassembler is able to identify the executed instructions and recover a disassembled version of the binary. Nonetheless, dynamic techniques have several weaknesses: (1) they only operate on the instructions that were executed in a particular set of runs. Therefore, only partial codes are disassembled; (2) program execution in a debug environment is slow and vulnerable to time-sensitive codes; (3) some instructions (e.g., exception handling) can not be analyzed correctly.

\subsection{Protection Method}

As it is believed to be impossible to completely prevent software cracking, software protection methods aim to make it sufficiently hard to understand the structure of a program. Hence, it is a practical challenge to protect the software from analysis and tampering to protect the proprietary algorithms and/or security critical codes. Presently, obfuscation, integrity verification and self-modifying codes (SMC) are the major anti-disassembly means.

Obfuscation technology [7] converts an original software into an equivalent form that crackers cannot easily understand. There are several software obfuscation methods such as fingerprinting [8], instruction occurrence [9], instruction re-ordering [10 and class transform [11. Particularly, in [12, with a one-way tamper-proof permutation, a point-function/boolean function such as password checking is obfuscated. These obfuscation technologies produce obfuscated software that is equivalent to the original software for all input. Nonetheless, based on control flow graph information and statistical methods, Kruegel et al. [13] presented binary analysis techniques which can identify a large fraction of the program's instructions. These analysis techniques substantially improve the success of the disassembly process when confronted with obfuscated binaries.

Integrity protection methods [14] 15] verify the code in real-time so as to prevent a tampered software from successfully running. Unfortunately, a substitution attack [16] 17] is applicable to all the integrity protections by modifying the underlying operating system. Although Giffin et al. [18] strengthened the checksum method with SMC code, they acknowledge that their improvement is vulnerable improvements in substitution attacks. Although control-flow integrity [19] is a way to enforce security, it is also naturally vulnerable to substitution attacks.

As a third protection method, SMC technology 21] alters software codes at the target addresses to produce the dynamic codes. As a special case of SMC, 
code encryption 22] - 25] scrambles the software, protecting the software from disassembling/tampering. Since the dynamic code generated by SMC technology is unknown in advance, a static disassembler cannot output a good assembly code. Thus it is difficult for the cracker to analyze and tamper the SMCprotected binary. Maebe et al. 26] has previously proposed to detect memory pages where SMCs occur utilizing the page protection mechanism of modern processors. However their implementation works for code run by a just-in-time compiler in a Linux environment, and hence reduces the performance of target software dramatically.

\subsection{Our Contribution}

Since the SMC-enabled static code structure is different from the dynamic code, the disassembled code may be incorrect if a static disassembler is used to analyze the static binary file. In order to produce a correct disassembly, the static disassembler should have access to static code which is the same as the runtime code. To this end, we remove the SMC protection using an exception mechanism that may occur during the execution of a Windows program. The attack disables the code modification attribute, triggering access violation exceptions each time a code modification is attempted. By intercepting the exception, we can obtain the modification's target address and codes, allowing us to perform the code modification. As a result, we can produce a static representation of the runtime code, in effect enhancing a static disassembler with some functions of a dynamic disassembler.

The outline of the present paper is as follows. Section 2 introduces the structure of the executable file and its mapping in the memory. Section 3 introduces SMC technology. Section 4 elaborates our proposal of removing the SMC. Section 5 proposes two implementations. Section 6 describes our experiments and results. We conclude in Section 7 .

\section{Primitives}

In this paper, we denote $[X]$ as the value stored in the address $X$ and $Y_{h}$ as the value $Y$ in hexadecimal.

\subsection{PE Structure}

The Portable Executable (PE) format [27] is a standard format under Microsoft Windows operating system. As a flat space structure, a PE-format executable is segmented into sections. Each section is a continuous structure of unlimited size but aligned along pag 11 boundaries. The PE header includes important information such as the address of the program entry point and the code section starting address. Each section header includes section attributes, e.g., READ

${ }^{1}$ A page is a continuous space of fixed size. For example, in Microsoft Windows XP, a page is of $4 \mathrm{~K}$ bytes in memory and 200 bytes in file. 
$\left(40000000_{h}\right)$, WRITE $\left(80000000_{h}\right)$, EXECUTE $\left(20000000_{h}\right)$.When a PE file is loaded into memory by a program loader, it is mapped into a real-time executable format. The structure in the memory is shown in Fig, 1, where the sections are

- .text: codes generated by the compiler or assembler.

- rdata: read-only data in run time.

- .data: initialization data.

- .idata: import table which includes other DLL (Dynamic Link Library) functions and re-localization information.

- .rsrc: resource data such as icons, menus, bitmaps etc.

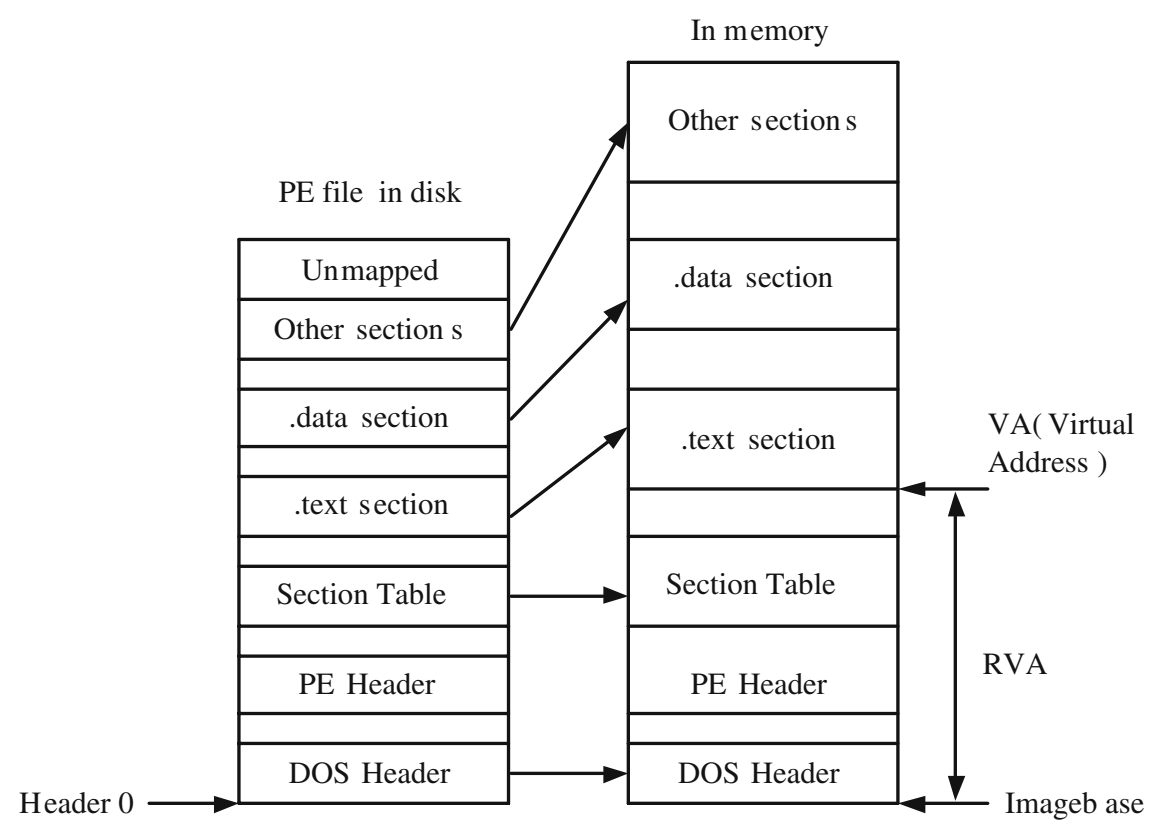

Fig. 1. PE file structure and mapping in memory

With regard to Fig, 1, each section in the memory maps to one section in the file. Suppose the base address of code section is $B_{f}$ in the file and $B_{m}$ in memory, then for any memory address $A_{m}$, its disk file location $A_{f}$ is

$$
A_{f}=B_{f}+\left(A_{m}-B_{m}\right)
$$

in the specific section. For the Microsoft Windows platform, the default base address is $B_{m}=400000_{h}$ for the code section. 


\section{2 try-except Mechanism}

The try-except statement is a Microsoft extension to the $\mathrm{C}$ and $\mathrm{C}++$ languages. It is a structured exception handler enabling 32-bit target applications to gain control when there are events that would normally terminate program execution. Such events are called exceptions which can be either hardware-based (e.g., access violation) or software-based (e.g., throw command). Exception handlers which process these exceptions as they occur are declared in the syntax shown in Fig,2, where the clause set $S_{1}$ is the body or guarded section where an exception might occur, and clause set $H_{1}$ is the exception handler.

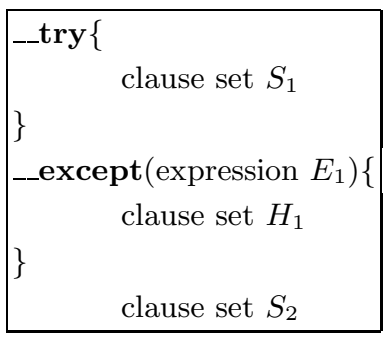

Fig. 2. try-except syntax

In the try-except mechanism, if no exception occurs during execution of the guarded section $S_{1}$ or its sub-routines, execution continues at the statement after the except clause, i.e., $S_{2}$. Otherwise, how the exception is handled is determined by the evaluation of the except expression $E_{1}$ :

- EXCEPTION_CONTINUE_EXECUTION (-1): Exception is dismissed. Program execution resumes at the point where the exception occurred.

- EXCEPTION_CONTINUE_SEARCH (0): Exception is not recognized. The program searches up the stack for a valid exception handler, first for containing try-except statements, then for the handler with the next highest precedence. If none is found, a system warning may occur as shown in Fig 3

- EXCEPTION_EXECUTE_HANDLER (1): Exception is recognized. Program control is transferred to the exception handler and the instructions in $H_{1}$ are executed to handle the exception. Thereafter program execution continues at $S_{2}$.

\section{Self-modifying Code}

\subsection{SMC Instruction Syntax}

In the following sections, we will illustrate the instructions with the x86 assembler language. For simplicity, we will focus on memory-write SMC instructions such as

$$
A_{1} \text { : opCode }\left[A_{2}\right], \text { src }
$$




\section{Microsoft Visual $C_{++}$}

Unhandled exception in test1.exe: 0xC0000005: Access Violation.

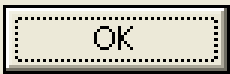

Fig. 3. Access Violation warning without a proper handler, where $C 0000005_{h}$ identifies the access violation exception

where opCode is the instruction code, $A_{1}$ is the address of the instruction, $A_{2}$ is the target address whose value will be changed by the SMC, and src is the target code to be written to address $A_{2}$. A list of possible SMC instructions is given in the Appendix. In this paper, we will use SMC to refer to the instruction which modifies the software code, and to refer to the whole code if there is no ambiguity.

Fig 4 illustrates an example where an instruction at address $A_{1}$ changes the code at address $A_{2}$ at run-time. Since the SMC modifies the code section, the instruction bytes present in the original executable is different from the actual instruction bytes executed at run-time.

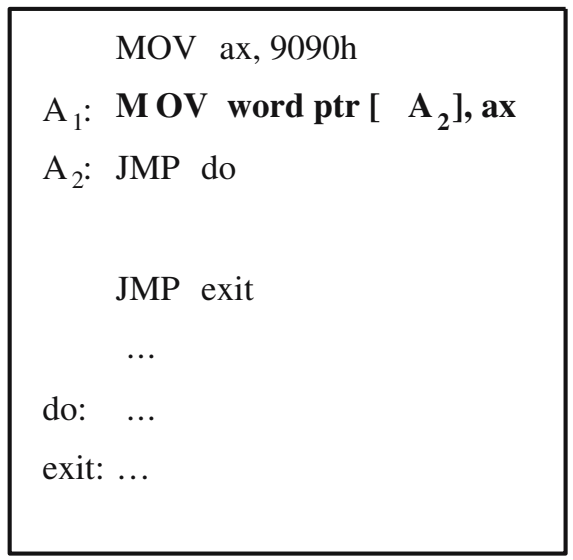

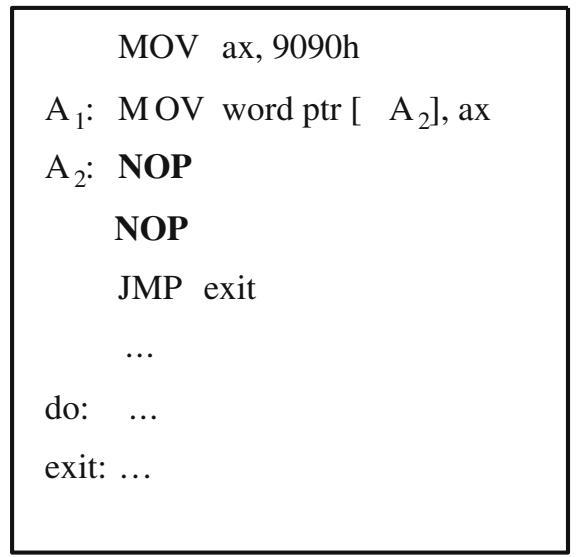

Fig. 4. Self-modifying code and its equivalent. The left side is the original program code, while the right side is the actual run-time code. Both sides are equal in function, where $90_{h}$ means "NOP"( no operation). 


\subsection{Violation by SMC}

To generate an executable, the compiler transforms source code (e.g., C/C++ code test1.cpp) into an object file (e.g., text1.obj). Following that, an executable (e.g., test1.exe) is generated with a linker. If the executable code is an SMC-enabled program without the required write permission, an access violation warning will occur as shown in Fig 5. Therefore, it is necessary to assign WRITE privilege to the target address to allow the SMC instruction to execute without exception.

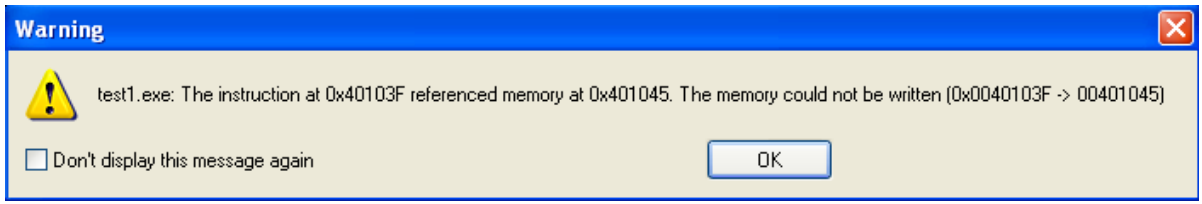

Fig. 5. Access violation warning when attempting a write to a non-writable code section in IDAPro environment, where $A_{1}=40103 F_{h}$ and $A_{2}=401045_{h}$

\subsection{Assigning WRITE Attribute}

In the Windows system, there are two ways to assign the WRITE attribute to the code in memory. One is to statically enable the whole code section to be writable with a linker. For instance, to generate a binary executable test 1 .exe from a text $1 . \mathrm{obj}$, perform

c>link/nologo/section.text, RWE test1.obj

where RWE means READ| WRITE| EXECUTE. The second way is to dynamically assign the WRITE attribute in real time with the API functions VirtualProtect() or VirtualProtectEX(). Since the second way enables us to change the WRITE attribute dynamically so as to deal with the access violation exception, we will elaborate the second method in Section 5

\section{Disassembling SMC-Enabled Executable}

According to the PE structure in Subsection 2.1, a software includes several sections which may be assigned different access attributes. While an SMC-enabled software may modify the code section as well as the data section, it is possible to differentiate data modification from code modification if we change the page attributes to produce access violation exceptions. From the data structure of the resulting exception, we are able to obtain the target address $A_{2}$ and target codes in an SMC-enabled code so as to disassemble the original software.

\subsection{Wrapping Original Software}

To obtain the target address of an SMC instruction, we control the execution of the target program $\mathbf{P}$. To this end, we produced a monitoring software $\mathbf{M}$ 
whose structure is as Fig, 6, where the program entry point (i.e., the address of the first executed instruction) of $\mathbf{P}$ is oldEntry. Hence, program $\mathbf{M}$ wraps up the original program $\mathbf{P}$ such that the original program $\mathbf{P}$ is the guarded code of try-except syntax in $\mathbf{M}$.

Denote the entire program as $\mathbf{M}_{P}$ which includes $\mathbf{M}$ and $\mathbf{P}$. Then the program entry point of $\mathbf{M}_{P}$ is the program entry point of $\mathbf{M}$. When $\mathbf{M}_{P}$ executes, $\mathbf{M}$ will call $\mathbf{P}$ with call oldEntry. Since program $\mathbf{M}$ and $\mathbf{P}$ are in the same address space, $\mathbf{M}$ can access the data/code of $\mathbf{P}$ such as the target address $A_{2}$ and value SMC.

\begin{tabular}{|c|c|}
\hline \multicolumn{2}{|c|}{ Merged program $\mathbf{M}_{P}$} \\
\hline Monitoring program $\mathbf{M}$ & Original program $\mathbf{P}$ \\
\hline$--\operatorname{try}\{$ & \\
\hline call OldEntry & OldEntry: \\
\hline$\}$ & $\vdots$ \\
\hline -_except $\left(E_{1}\right)\{$ & $A_{1}:$ opCode $\left[A_{2}\right], \operatorname{src}(\mathrm{SMC})$ \\
\hline exception handler $H_{1}$ & $\vdots$ \\
\hline$\}$ & \\
\hline
\end{tabular}

Fig. 6. Wrapping the original program

\subsection{Locating the SMC Code}

As SMCs essentially perform write operations to a location in memory, an access violation will occur if the SMC attempts to write bytes to a non-writable address $A_{2}$. Therefore if an adversary alters the entire code section to be non-writable, access violation exceptions will occur whenever SMCs in $\mathbf{M}_{P}$ are executed. From the exception structure, the adversary can obtain the address where the violation occurs and thus the target address and code, defeating the SMC protection. Specifically, after program $\mathbf{M}_{P}$ is started,

1. Program control is transferred to the guarded section, i.e., the program entry point oldEntry. Program $\mathbf{P}$ will execute normally until an exception occurs.

2. If the exception is handled by $\mathbf{P}$ itself, execution continues without control being transferred to $\mathbf{M}$.

3. Otherwise, exception handling is passed to $\mathbf{M}$. If the exception that occurred is an EXCEPTION_ACCESS_VIOLATION exception, $\mathbf{M}$ will record the SMC that attempted to execute, perform the SMC, then allow $\mathbf{P}$ to resume at the next instruction.

\subsection{Disassembling Target Code}

After locating the SMC codes, the adversary obtains a log file containing the SMC codes that $\mathbf{P}$ attempted to execute and their address in memory. Based on 
the mapping rule between memory and file locations, the adversary can modify the executable file with Eq.(11) to generate an equivalent executable with the modifications that would have been performed by the SMC. From this executable, a disassembler such as IDAPro can obtain an accurate static disassembly.

\section{Implementations}

This section describes two implementations using the Windows XP platform with $\mathrm{C}++$ programming language. The first implementation wraps the target software to form a merged program, while the second one debugs the target software. Both methods are able to extract the necessary data: the target address and target code of the SMC. The following Subsections 5.1 5.5 elaborate the first implementation, and Subsection 5.6 describes the second implementation.

\subsection{Creating Program M}

As shown in Fig 7 program $\mathbf{M}$ includes two modules: filter $(\cdot)$ which determines the SMC and clearWR(.) which performs the SMC so that $\mathbf{P}$ executes properly.

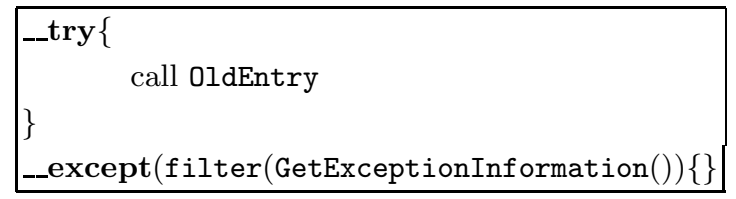

Fig. 7. Structure of program M

filter When an exception occurs, filter receives an exception structure from the OS which includes the exception code, exception address $A_{1}$, etc. If the exception code is EXCEPTION_ACCESS_VIOLATION $\left(C 0000005_{h}\right)$, the filter routine will:

- Extract the SMC address $A_{1}$ from the exception structure, and read several (e.g., 128) code bytes starting from $A_{1}$ into a string $S$.

- Parse $S$ to obtain the SMC instruction according to Table 1 .

- Assign the pages including $\left[A_{1}, A_{1}+n\right)$ with the WRITE attribute, where $n$ is the size of of the SMC instruction.

- Save $S$ into a buffer $\mathbb{B}$.

- Replace the bytes in address $\left[A_{1}, A_{1}+n\right)$ with instruction code "call clearWR", filling any excess bytes with NOP instructions.

- Return the value -1 , instructing the program to resume execution at the point where the exception occurred.

For all other exception codes, filter returns the value 0 to instruct the program to continue searching up the stack for an appropriate handler. We assume $n$ is at least the size of the call instruction. If not, we can always parse the instruction following the SMC and save that instruction to $\mathbb{B}$ as well. 
clearWR clearWR operates as follows.

- Obtain the SMC from the saved buffer $\mathbb{B}$ and re-write it back to its original location

- Parse the SMC instruction. Assume the region $\left[A_{2}, A_{2}+m\right)$ will be written by SMC, where $m$ is the size of written region.

- Assign WRITE attribute to the pages that cover address region $\left[A_{2}, A_{2}+m\right)$ exactly.

- Execute the SMC code in the address space of $\mathbf{M}$.

- Disable the WRITE attribute of all the pages that cover address region $\left[A_{1}, A_{1}+n\right) \cup\left[A_{2}, A_{2}+m\right)$ exactly.

- Record $A_{2}$ and the new bytes in the region $\left[A_{2}, A_{2}+m\right)$ into a log file.

- Return program control to $\mathbf{P}$.

filter cannot perform the SMC directly since by the exception handling mechanism, program execution will either continue at where the exception occurred, i.e., the SMC code, or after the except clause, i.e., the end of the program $\mathbf{M}$. Thus by inserting the instruction call clearWR at the address $A_{1}$, clearWR will be performed instead of the SMC after filter returns. Subsequently after clearWR returns, the instruction pointer can move to the next instruction.

In clearWR, the SMC is restored to its original location so that program $\mathbf{P}$ can be run correctly even if the SMC is included in an iterative structure or protected by a checksum-like mechanism.

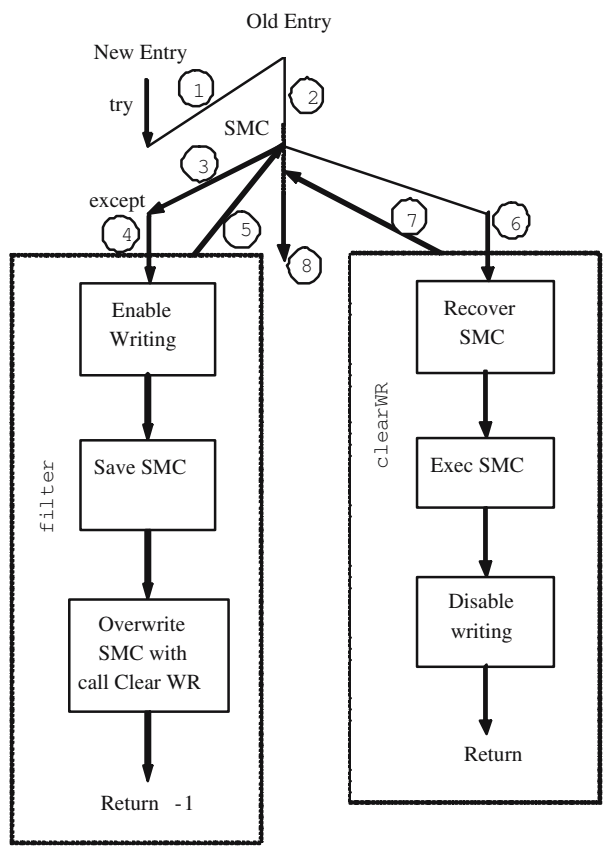

Fig. 8. Program control flow when an SMC occurs 


\subsection{Designing Program Structure}

As mentioned in Section 4, we modify the original program $\mathbf{P}$ such that the monitoring program $\mathbf{M}$ can control $\mathbf{P}$. We generate the new program structure as Fig.7. When an exception handling is passed to $\mathbf{M}$, the program control will be passed to the exception filter, and GetExceptionInformation(.) returns the exception structure. The new program control flow will occur as shown in Fig 8 .

\subsection{Modifying WRITE Attribute}

In a protected software, there may be several SMC instructions randomly located in the program $\mathbf{P}$. In order to detect all the SMC instructions, an adversary will remove the WRITE attribute of the code section, but assign the WRITE attribute to the target address so that the program $\mathbf{P}$ runs correctly. To this end, the adversary adopts the attribute assignment functions:

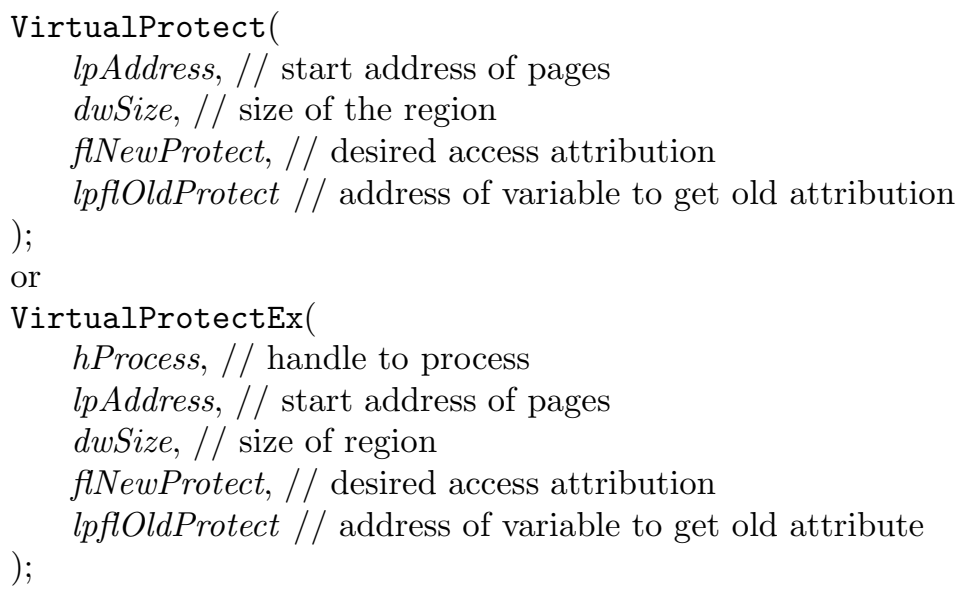

These two functions assign the attribute flNewProtect=EXECUTE_READ_ WRITE to pages including the specified address [lpAddress, lpAddress + dwSize).

For each access violation exception, we change the page that includes the target address to be writable with one of the above instructions. As a result, the SMC can be executed properly.

\subsection{Integrating Codes}

After producing the monitoring program $\mathbf{M}$, the adversary merges it with the original program $\mathbf{P}$. The integrating process is as follows.

- Change the attribute of the code section of $\mathbf{P}$ to EXECUTE_READ only.

- Add a new section with address rva which is beyond the address space of P.

- Execute the linker command as 
- Copy the code section of the monitoring program $\mathbf{M}$ into the new blank section.

- Change the program entry AddressofEntryPoint to that of the new program $\mathbf{M}$.

- Insert functions such as VirtualProtect, Virtualquery and RaiseException into the import table.

\subsection{Detecting Craft Code}

If the original program $\mathbf{P}$ uses the same method to enable SMC, the present method may not work since access violation exceptions are handled by program $\mathbf{P}$ itself. To overcome this weakness, we can detect the function VirtualProtect from the import table, and change the attribute parameter flNewProtect back to non-writable such that $\mathbf{P}$ will not respond to access violation exceptions.

Additionally, in the Subsection 4.3. we only considered cases that SMC replaces dummy code in region $\left[A_{2}, A_{2}+m\right.$ ). If the code in the region is useful, (i.e., the same address is used for two more instructions, for example encrypted code), we should enable the disassembler to disassemble both the old code and the target code. That is to say, the present method can be extended to disassemble encrypted codes.

\subsection{Alternative Implementation}

Following Section 4, the task of the monitoring program includes the steps: disabling WRITE attribute of the SMC's target address, intercepting the SMC instruction, restoring the WRITE attribute, and finally executing SMC. Hence, if we build a debugging environment such that SMC can be executed by SingleStep, we are able to find the target address and target code too. Fig 9 illustrates this debugger-like implementation. In this alternative implementation, the monitor program $\mathbf{T}$

(1) Disables the WRITE attribute of the code section of $\mathbf{P}$, then loads and runs P.

(2) Wait for an access violation from $\mathbf{P}$. When an access violation exception occurs, the exception handler in $\mathbf{T}$ will parse the SMC and obtain the target address.

(3) Enable the WRITE attribute of the target address, and initiate SINGLE_ STEP interruption.

(4) Execute SMC in Single-step mode, and activate single-step exception.

(5) Remove WRITE attribute of target address via the EXCEPTION_SINGLE_ STEP exception handler.

(6) Recode the target address and target code.

In comparison with the previous wrapper implementation, this method can process the craft code in Subsection 5.5 by intercepting access violation exceptions before the program's own exception handling routine. However, this method takes more computation time since an EXCEPTION_SINGLE_STEP exception and debugging operation are processed for each SMC execution. 


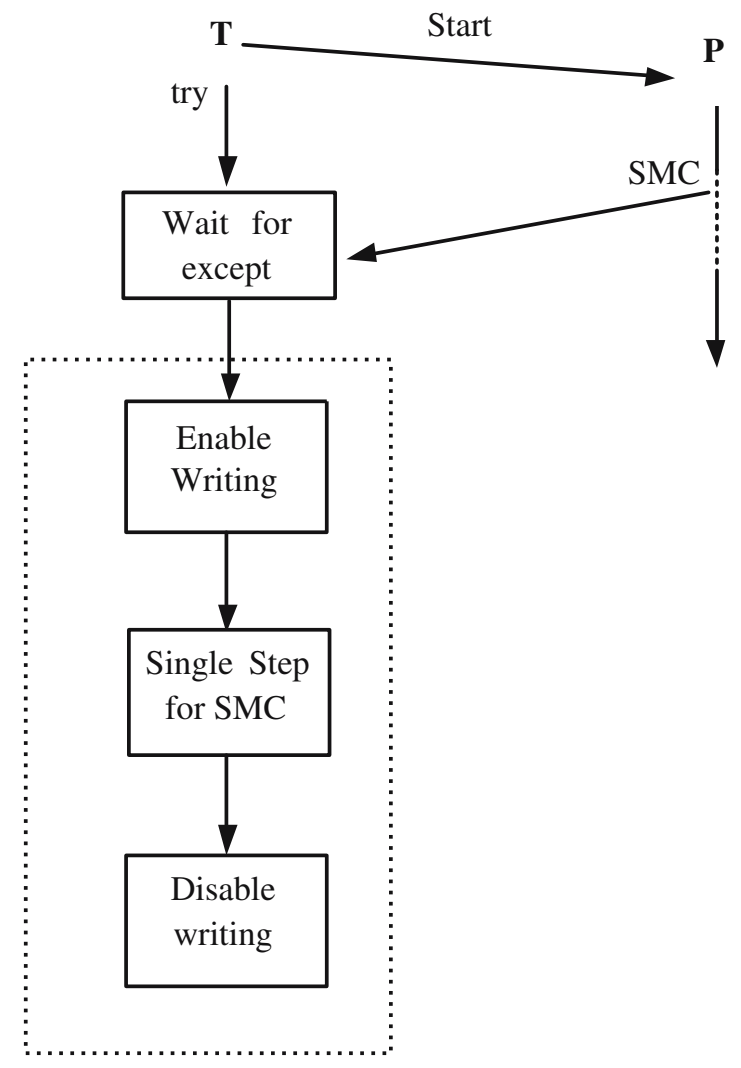

Fig. 9. Debugger-like implementation

\section{Experiments}

\subsection{Improvement on Disassembler}

In this experiment, we create a sample binary executable and used the disassembler IDAPro for test tool. Fig 10(a) is the disassembly code generated with IDAPro directly. With the proposed method, the monitoring program outputs the target address and target code in the SMC instructions, and records them. After modifying the SMC-enabled code with the recorded data, we disassemble the modified code with IDAPro.exe again, the new disassembly code is shown in Fig 10(b). Clearly, the wrapper-assisted disassembler outputs a better assembly code in case of SMC.

\subsection{Time Overhead}

In our scheme, since the exception of $\mathbf{P}$ is processed in the monitoring program $\mathbf{M}$, the run time of $\mathbf{P}$ will be increased. To evaluate the time cost, the freeware gzip 


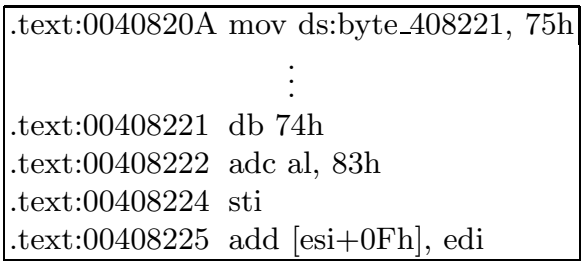

(a) Output of original disassembler.

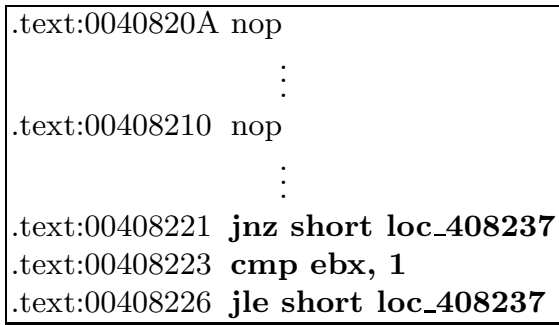

(b) Output of enhanced disassembler.

Fig. 10. Output difference between original disassembler and enhanced disassembler. After detecting and accounting for the code modification performed at $A_{1}=40820 A$, we can obtain an accurate disassembly shown in (b).

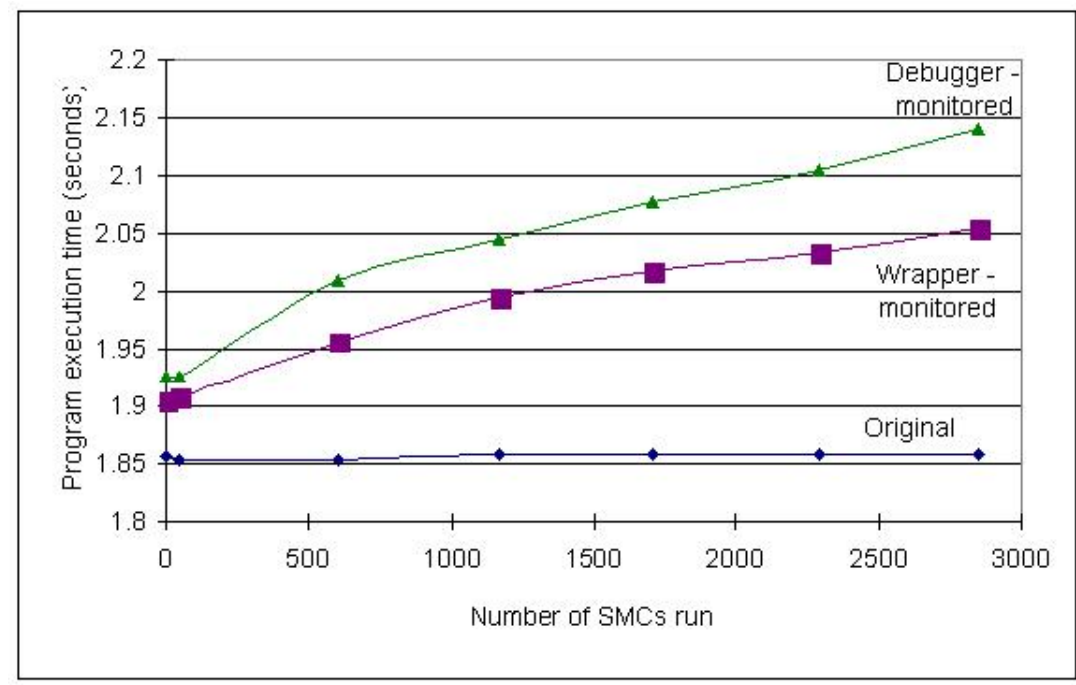

Fig. 11. Overhead of Execution time. The lower, middle and upper curves describe the time used in original gzip, wrapper-monitored gzip and debugger-monitored gzip respectively.

package 28] is used as a tested sample. We inserted a number of SMC instructions into the protected program gzip, and calculated the time taken to compress a $16 \mathrm{MB}$ collection of text files. Fig[1] shows the time cost with regard to the number of SMC instructions executed. Generally, the time cost is only increased $10 \%$, or $35 \mu s$ per SMC instruction using a Pentium IV $2.2 \mathrm{GHz}$ system. Analysis can also be restricted to a targeted code subsection by disabling the WRITE attribute only for that subsection. Hence, the proposed scheme can detect the SMC easily with little time cost. According to Fig 11, the debugger-like implementation consumes 
more time. In fact, this observation is sound since an extra interruption and singlestep exception are executed for each SMC in a debug environment.

\section{Conclusion and Future Work}

SMC changes the software in real-time such that the dynamic code is different from the static code, and hence provides an effective way to defeat static disassembler. However, if a monitoring program identifies the target address of the SMC codes and replaces the bytes in the target addresses with target bytes, it will produce a corrected static code which is identical to dynamic code. This paper presents a method which employs exception mechanism, and implements the method in two implementations. Our experiments demonstrate that the proposed method is effective in defeating SMC protection.

The program can counter this attack by regularly enabling the write privilege for its code section using methods other than the functions mentioned in Subsection 5.3. though this would require additional execution time. However, we should be able to determine these methods and devise similar counters.

\section{References}

1. Gavin Clarke, "DVD Jon Hacks Media Player File Encryption," Sept. 02, 2005, (http://www.theregister.co.uk/2005/09/02/dvd_jon_mediaplayer/

2. IDA Pro Technologies \& Features Highlights, http://www.datarescue.com/idabase/technologies.htm

3. MicroAPL Porting Tools, http://www.microapl.co.uk/Porting/index.html

4. Free Software Foundation. GNU Binary Utilities, Mar 2002. http://www.gnu.org/software/binutils/manual/

5. B. Schwarz, S. Debray, and G. Andrews, "Disassembly of executable code revisited.," 9th Working Conference on Reverse Engineering, pp. 45C54, 2002.

6. rordbg, http://bbs.pediy.com/upload/2006/8/files/rordbg.rar_116.rar

7. C. Linn and S. Debray, "Obfuscation of executable code to improve resistance to static disassembly.," 10th ACM Conference on Computer and Communications Security (CCS), pp.290-299, 2003.

8. R. L. Davidson, N. Myhrvold, "Method and System for Generating and Auditing a Signature for a Computer Program," US Patent 5,559,884, Assignee: Microsoft Corp, 1996.

9. J.P. Stern, G. Hachez, F. Koeune, J.-J. Quisquater, "Robust Object Watermarking: Application to Code," 3rd Workshop on Information Hiding, LNCS 1768, pp.368378, 1999

10. Masahiro Mambo, Takanori Murayama, Eiji Okamoto, "A Tentative Approach to Constructing Tamper-Resistant Software," 1997 New Security Paradigms Workshop, pp.23-33.

11. Mikhail Sosonkin, Gleb Naumovich, Nasir Memon, "Obfuscation of Design Intent in Object-oriented Applications," ACM Workshop On Digital Rights Management, pp.142-153, 2003.

12. Hoeteck Wee, "On Obfuscating Point Functions," Annual ACM symposium on Theory of computing (STOC), pp. 523-532 2005. 
13. Christopher Kruegel, William Robertson, Fredrik Valeur, and Giovanni Vigna, "Static Disassembly of Obfuscated Binaries," USENIX security Symposium, pp.255-270, 2005.

14. H. Chang and M. Atallah, "Protecting Software Code by Guards," Security and Privacy in Digital Rights Management, LNCS 2320, pp.160-175, 2001.

15. Bill Horne, Lesley R. Matheson, Casey Sheehan, Robert Endre Tarjan, "Dynamic Self-Checking Techniques for Improved Tamper Resistance," Digital Rights Management, LNCS 2320, pp.141-159, 2001.

16. G.Wurster, P. C. van Oorschot, and A. Somayaji, "A Generic Attack on Checksumming-based Software Tamper Resistance," IEEE Symposium on Security and Privacy, pp.127-138, 2005.

17. P. C. van Oorschot, A. Somayaji, and G.Wurster, "Hardware assisted circumvention of self-hashing software tamper resistance," IEEE Transactions on Dependable and Secure Computing, 2(2):82-92, 2005.

18. Jonathon T. Giffin, Mihai Christodorescu, Louis Kruger, "Strengthening Software Self-Checksumming via Self-Modifying Code," pp.23-32, 21st Annual Computer Security Applications Conference, 2005. http://www.cs.wisc.edu/wisa/papers/acsac05/GCK05.pdf

19. Martin Abadi, Mihai Budiu, Ulfar Erlingsson, and Jay Ligatti, "Control-flow integrity: Principles, Implementations, and Applications," ACM conference on Computer and communications security, pp.340-353, 2005.

20. M. Christodorescu, and Somesh Jha, "Static Analysis of Executables to Detect Malicious Patterns," USENIX Security Symposium, pp.169-186, 2003.

21. Yuichiro Kanzaki, Akito Monden, Masahide Nakamura, Ken-ichi Matsumoto, "Exploiting Self-Modification Mechanism for Program Protection," International Computer Software and Applications Conference (COMPSAC), pp.170-179, 2003

22. D. J. Albert and S. P. Morse, "Combating Software Piracy by Encryption and Key Management," Computer, Apr. 1984.

23. D. W. Aucsmith, "Tamper Resistant Software: An Implementation," Information Hiding Workshop, LNCS 1174, pp.317-333, 1996.

24. Ping Wang, Tamper Resistance for Software Protection, Master Thesis, Information and Communications University, Korea, 2005.

25. Jaewon Lee, Heeyoul Kim, and Hyunsoo Yoon, "Tamper Resistant Software by Integrity-Based Encryption," PDCAT 2004, LNCS 3320, pp. 608C612, 2004.

26. Jonas Maebe, Koen De Bosschere, "Instrumenting Self-Modifying Code," Fifth Intl. Workshop on Automated and Algorithmic Debugging, pp. 103-113, Sep. 2003.

27. Microsoft Corporation, "Microsoft Portable Executable and Common Object File Format Specification", Revision 6.0, February 1999, http://www.microsoft.com/whdc/system/platform/firmware/PECOFF.mspx

28. The gzip compression program, http://www.gzip.org/

\section{A SMC Instructions}

Table 1 lists possible SMC instructions where $A_{2}$ and/or src may be stated implicitly in some instructions. 
Table 1. SMC instructions

\begin{tabular}{|c|c|c|c|}
\hline Instruction & opCode & semantics & size \\
\hline $\mathrm{ADD}(\mathrm{ADC}) \mathrm{mem}, \mathrm{reg}$ & $01(11) / \mathrm{r}$ & Add (with CF) r32 to r/m32 & 6 \\
\hline ADD mem, imm & $81 / 0(2)$ id & Add (with CF) imm32 to $\mathrm{r} / \mathrm{m} 32$ & 10 \\
\hline SUB mem, reg & $29 / \mathrm{r}$ & Subtract r32 from $\mathrm{r} / \mathrm{m} 32$ & 6 \\
\hline SUB mem, imm & $81 / 5 \mathrm{id}$ & Subtract imm32 from $\mathrm{r} / \mathrm{m} 32$ & 10 \\
\hline DEC(INC) mem & $\mathrm{FF} / 1(0)$ & Decrement(increment) r/m32 & 6 \\
\hline AND mem, reg & $21 / \mathrm{r}$ & AND r32 to $\mathrm{r} / \mathrm{m} 32$ & 6 \\
\hline AND mem, imm & $81 / 4 \mathrm{id}$ & AND imm32 to r $/ \mathrm{m} 32$ & 10 \\
\hline OR(XOR) mem, reg & $09(31) / \mathrm{r}$ & OR(XOR) r32 to $\mathrm{r} / \mathrm{m} 32$ & 6 \\
\hline OR(XOR) mem, imm & $81 / 1(6)$ id & OR(XOR) imm32 to $\mathrm{r} / \mathrm{m} 32$ & 10 \\
\hline NEG mem & F7 /3 & 2's complement negate $\mathrm{r} / \mathrm{m} 32$ & 6 \\
\hline NOT mem & F7 /2 & Reverse each bit of $\mathrm{r} / \mathrm{m} 32$ & 6 \\
\hline POP mem & $8 \mathrm{~F} / 0$ & Pop stack into mem32 & 6 \\
\hline MOV mem, reg & $89 / \mathrm{r}$ & Move r32 to $\mathrm{r} / \mathrm{m} 32$ & 6 \\
\hline MOV mem, imm & $\mathrm{C} 7 / 0$ & Move imm32 to $\mathrm{r} / \mathrm{m} 32$ & 10 \\
\hline MOV mem16, segreg & $8 \mathrm{C} / \mathrm{r}$ & Move segment reg to $\mathrm{r} / \mathrm{m} 16$ & 2 \\
\hline $\mathrm{MOVS} / / \mathrm{B} / \mathrm{W} / \mathrm{D}$ & $\mathrm{A} 4(5)$ & Move from DS:ESI to ES:EDI & 1 \\
\hline STOS mem & $\mathrm{AB}$ & Store EAX at ES:EDI & 1 \\
\hline XADD mem, reg & $0 \mathrm{~F} \mathrm{C} 1 / \mathrm{r}$ & $\begin{array}{l}\text { Exchange r32 and } \mathrm{r} / \mathrm{m} 32 \\
\text { Store sum in } \mathrm{r} / \mathrm{m} 32\end{array}$ & 7 \\
\hline XCHG mem, reg & $87 / \mathrm{r}$ & Exchange r32 with $\mathrm{r} / \mathrm{m} 32$ & 6 \\
\hline RCL(RCR) mem, imm8 & $\mathrm{C} 1 / 2(3) \mathrm{ib}$ & Rotate(CF) left(right) imm8 times & 7 \\
\hline RCL(RCR) mem, CL & D3 /2(3) & Rotate(CF) left(right) imm8 times & 6 \\
\hline ROL(ROR) mem, imm8 & $\mathrm{C} 1 / 0(1)$ & Rotate left(right) imm8 times & 7 \\
\hline ROL(ROR) mem, CL & D3 /0(1) & Rotate left(right) imm8 times & 6 \\
\hline SHL(SHR) mem, imm8 & C1 /4(5) & Mult(div) by 2, imm 8 times & 7 \\
\hline SHL(SHR) mem, CL & D3 /4(5) & Mult(div) by 2, imm8 times & 6 \\
\hline SAL/SAR mem, imm8 & C1 /4(7) & Signed mult(div) by 2, imm 8 times & 7 \\
\hline SAL/SAR mem, CL & D3 /4(7) & Signed mult(div) by 2, imm8 times & 6 \\
\hline SHLD(SHRD) mem, reg, CL & $0 \mathrm{~F}$ A5(D) & $\begin{array}{l}\text { Shift r/m32 CL places left(right) } \\
\text { and shift bits in from r32 }\end{array}$ & 7 \\
\hline SHLD(SHRD) mem, reg, imm8 & $0 \mathrm{~F} \mathrm{~A} 4(\mathrm{C})$ & $\begin{array}{l}\text { Shift } \mathrm{r} / \mathrm{m} 32 \text { imm } 8 \text { places left(right) } \\
\text { and shift bits in from } \mathrm{r} 32\end{array}$ & 7 \\
\hline
\end{tabular}

